\title{
On the instability of the solutions of some nonlinear vector differential equations of fourth order
}

\author{
Cemil Tunç
}




\title{
ON THE INSTABILITY OF THE SOLUTIONS OF SOME NONLINEAR VECTOR DIFFERENTIAL EQUATIONS OF FOURTH ORDER
}

\author{
CEMIL TUNÇ
}

Received 23 December, 2009

\begin{abstract}
The purpose of this paper is to obtain some new sufficient conditions which guarantee the instability of the trivial solution of some certain nonlinear vector differential equations of fourth order. Our results improve and include some well known instability results, which were established on the instability of the trivial solution of certain scalar differential equations of fourth order, to certain nonlinear vector differential equations of fourth order.
\end{abstract}

2000 Mathematics Subject Classification: 34D05, 34D20

Keywords: nonlinear differential equations, fourth order, instability, the Lyapunov's second (or direct) method.

\section{INTRODUCTION}

Consider the following nonlinear vector differential equations

$$
x^{(4)}+A \dddot{x}+G(x, \dot{x}, \ddot{x}, \dddot{x}) \ddot{x}+H(x) \dot{x}+F(x, \dot{x}, \ddot{x}, \dddot{x}) x=0
$$

and

$$
x^{(4)}+\Phi(\dddot{x})+\Psi(x, \dot{x}, \ddot{x}, \dddot{x}) \ddot{x}+C \dot{x}+D x=0,
$$

where $A, C$ and $D$ are constant symmetric $n \times n$ - matrices; $G, H, F$ and $\Psi$ are continuous (symmetric $n \times n$-) matrix valued functions; $\Phi: R^{n} \rightarrow R^{n}, \Phi(0)=0$ and $\Phi$ is continuous. Throughout this paper, instead of equations (1.1) and (1.2), we consider the system of differential equations:

$$
\begin{aligned}
\dot{x} & =y, \dot{y}=z, \dot{z}=w, \\
\dot{w} & =-A w-G(x, y, z, w) z-H(x) y-F(x, y, z, w) x
\end{aligned}
$$

and

$$
\begin{aligned}
& \dot{x}=y, \dot{y}=z, \dot{z}=w, \\
& \dot{w}=-\Phi(w)-\Psi(x, y, z, w) z-C y-D x,
\end{aligned}
$$

which were obtained as usual by setting $\dot{x}=y, \ddot{x}=z, \dddot{x}=w$ from equations (1.1) and (1.2), respectively. 
Now, let $J(H(x) x \mid x)$ and $J_{\Phi}(w)$, respectively, denote the linear operators from the matrix $H(x)$ and gradient vector field $\Phi(w)$ to the matrices

$$
J(H(x) x \mid x)=\left(\frac{\partial}{\partial x_{j}} \sum_{k=1}^{n} h_{i k} x_{k}\right)=H(x)+\left(\sum_{k=1}^{n} \frac{\partial h_{i k}}{\partial x_{j}} x_{k}\right)
$$

and

$$
J_{\Phi}(w)=\left(\frac{\partial \phi_{i}}{\partial w_{j}}\right),(i, j=1,2, \ldots, n),
$$

where $\left(x_{1}, x_{2}, \ldots, x_{n}\right),\left(w_{1}, w_{2}, \ldots, w_{n}\right),\left(h_{i k}\right)$ and $\left(\phi_{1}, \phi_{2}, \ldots, \phi_{n}\right)$ are components of $x, w, H$ and $\Phi$, respectively. Further, it is assumed that the matrices $J(H(x) x \mid x)$ and $J_{\Phi}(w)$ exist and are symmetric and continuous.

The unstable behavior of solutions for various certain scalar and vector linear and nonlinear differential equations of fourth order has been discussed in the literature over the past three decades, and they are still being investigated. We refer the reader to the papers of Ezeilo [2-4], Sadek [7], Skrapek [8], Tunç [9-11] and the references thereof for some publications performed on the subject.

It should be noted that, in 2000, Ezeilo [3] considered the following fourth order scalar nonlinear differential equations

$$
x^{(4)}+a_{1} \dddot{x}+g(x, \dot{x}, \ddot{x}, \dddot{x}) \ddot{x}+h(x) \dot{x}+f(x, \dot{x}, \ddot{x}, \dddot{x})=0
$$

and

$$
x^{(4)}+p(\dddot{x}, \ddot{x})+q(x, \dot{x}, \ddot{x}, \dddot{x}) \ddot{x}+a_{3} \dot{x}+a_{4} x=0,
$$

respectively, where $a_{1}, a_{3}$ and $a_{4}$ are constants. He proved two theorems on the instability of the trivial solution, $x=0$, of equations (1.5) and (1.6). To the best of our knowledge, so far, neither the instability of solutions of nonlinear vector differential equations of the form (1.1) and (1.2) has been investigated in the literature nor papers have been published which were based on the results of Ezeilo [3]. When $f(x, \dot{x}, \ddot{x}, \dddot{x})=F(x, \dot{x}, \ddot{x}, \dddot{x}) x$ in (1.5) and $p(\dddot{x}, \ddot{x})=\Phi(\dddot{x})$ in (1.6), our problems, which will be related to equations (1.1) and (1.2), improve and include the problems discussed by Ezeilo [3] from the scalar cases to the vector cases, respectively. It is also worth mentioning that throughout the aforementioned papers, based on Krasovskii's criterion (see Krasovskii [5]), the Lyapunov's [6] second (or direct) method has been used as a basic tool to prove the results established thereof. In this paper, we utilize the same method to verify our main results. It is also well known that, by now, the Lyapunov's [6] second (or direct) method is the most effective tool to study the stability and instability of solutions of nonlinear differential equations of higher order, when there is no analytical expression for solutions. The motivation for the present work has been inspired basically by the paper of Ezeilo [3], Tunç [11] and the papers mentioned above. Finally, all the papers aforementioned have been published without including an example on the topic, except that of Sadek [7] and Tunç [11]. In this paper, we also give an example for the illustration of the subject. 
We now consider the constant-coefficient fourth order scalar differential equation

$$
x^{(4)}+a_{1} \dddot{x}+a_{2} \ddot{x}+a_{3} \dot{x}+a_{4} x=0 .
$$

We know from the qualitative theory of ordinary differential equations that the trivial solution of the above equation is unstable if and only if the associated (auxiliary) equation

$$
\psi(r):=r^{4}+a_{1} r^{3}+a_{2} r^{2}+a_{3} r+a_{4}=0
$$

has at least one root with a positive real part. Naturally, the existence of such a root depends on (though not always all of) the coefficients $a_{1}, a_{2}, a_{3}$ and $a_{4}$ of $\psi$. The corresponding auxiliary equation $\psi(r)=0$ of the above equation has no purely imaginary roots $r=i \lambda,(\lambda \neq 0)$ if

$$
\chi_{1}:=\lambda^{4}-a_{2} \lambda^{2}+a_{4} \neq 0
$$

or if

$$
\chi_{2}:=a_{1} \lambda^{2}-a_{3} \neq 0 .
$$

Note that $\chi_{1}$ may be rearranged in the form

$$
\chi_{1}=\left(\lambda^{2}-\frac{1}{2} a_{2}\right)^{2}+a_{4}-\frac{1}{2} a_{2}^{2}
$$

so that $\chi_{1} \neq 0$ holds if, for example, $a_{4}>\frac{1}{4} a_{2}^{2}$. It is also easy to see that $\chi_{2} \neq 0$ will hold if

$$
a_{1} a_{3}<0, a_{4} \neq 0,
$$

where the condition on $a_{4}$ here is necessary in order to ensure that $\lambda \neq 0$. It should also be noted that it was shown in Ezeilo [2] that the existence of the relation $a_{4}>$ $\frac{1}{4} a_{2}^{2}$ between the coefficients $a_{2}$ and $a_{4}$ of the equation is a sufficient condition that guarantees that the solution $x=0$ of aforementioned equation is unstable. The above preamble is merely intended to give the background to the assumptions which play a dominant role in our treatment here and to give some indication of how these assumptions stand with respect to the Routh-Hurwitz criteria.

Throughout the paper, the symbol $\langle x, y\rangle$ is used to denote the usual scalar product in $R^{n}$ for given any $x, y$ in $R^{n}$, that is, $\langle x, y\rangle=\sum_{i=1}^{n} x_{i} y_{i}$, thus $\|x\|^{2}=\langle x, x\rangle$. It is also well known that a real symmetric matrix $A=\left(a_{i j}\right),(i, j=1,2, \ldots, n)$ is said to be positive definite if and only if the quadratic form $Q:=x^{T} A x\left(x \in R^{n}\right)$ is positive definite.

\section{MAIN RESUlTS}

We first state the following algebraic result, which will be useful in the proof of the main results 
Lemma 1. Let $A$ be a real symmetric $n \times n$-matrix and

$$
a^{\prime} \geq \lambda_{i}(A) \geq a>0,(i=1,2, \ldots, n),
$$

where $a^{\prime}$ and a are constants, resp. $\lambda_{i}(A)$ denotes the $i-t$ th eigenvalue of $A$. Then

$$
a^{\prime}\langle x, x\rangle \geq\langle A x, x\rangle \geq a\langle x, x\rangle
$$

and

$$
a^{\prime 2}\langle x, x\rangle \geq\langle A x, A x\rangle \geq a^{2}\langle x, x\rangle .
$$

Proof. See Bellman [1].

Our first result is the following theorem

Theorem 1. In addition to the basic assumptions imposed on $A, G, H$ and $F$ that appear in equation (1.1), we suppose that there exists a constant $a_{1}$ such that the following conditions hold:

$$
\lambda_{i}(A) \geq a_{1}>0
$$

and

$$
\lambda_{i}(F(x, y, z, w,))>\frac{1}{4}\left[\lambda_{i}(G(x, y, z, w))\right]^{2},(i=1,2, \ldots, n),
$$

for all $x, y, z, w \in R^{n}$.

Then the trivial solution $x=0$ of equation (1.1) is unstable for arbitrary $H(x)$.

Remark 1. In order to prove Theorem 1, it will be sufficient to show that there exists a continuous Lyapunov function $V=V(x, y, z, w)$ which has the following Krasovskii properties (see Krasovskii [5]):

$\left(K_{1}\right)$ In every neighborhood of $(0,0,0,0)$, there exists a point $(\xi, \eta, \zeta, \mu)$ such that $V(\xi, \eta, \zeta, \mu)>0$

$\left(K_{2}\right)$ the time derivative $\dot{V}$ along solution paths of the system (1.3) is positive semi-definite; and

$\left(K_{3}\right)$ the only solution of the system (1.3) which satisfies $\dot{V}(t)=0,(t \geq 0)$, is the trivial solution $(0,0,0,0)$.

Remark 2. It should be noted that there is no restriction on the eigenvalues of the matrix $H$ in (1.3).

Remark 3. Our first result, Theorem 1, includes the result of Ezeilo [3, Theorem 1] and Sadek [7, Theorem 1], and improves the result of Ezeilo [3, Theorem 1] from scalar differential equation to the vector differential equation, (1.1). Equation (1.1) is also completely different from those studied in the literature, (see also Ezeilo [2-4], Sadek [7], Skrapek [8] and Tunç [9-11]. 
Proof. For the proof of Theorem 1, we define a Lyapunov function $V_{0}=V_{0}(x, y, z, w)$ given by:

$$
V_{0}=-\langle x, w\rangle+\langle y, z\rangle-\langle x, A z\rangle+\frac{1}{2}\langle A y, y\rangle-\int_{0}^{1}\langle H(\sigma x) x, x\rangle d \sigma .
$$

Observe that $V_{0}(0,0,0,0)=0$. Indeed, in view of the assumptions of Theorem 1, we have

$$
\begin{aligned}
V_{0}(0, \varepsilon, \varepsilon, \varepsilon) & =\langle\varepsilon, \varepsilon\rangle+\frac{1}{2}\langle A \varepsilon, \varepsilon\rangle \\
& \geq\langle\varepsilon, \varepsilon\rangle+\frac{1}{2} a_{1}\langle\varepsilon, \varepsilon\rangle \\
& =\|\varepsilon\|^{2}+\frac{1}{2} a_{1}\|\varepsilon\|^{2}>0
\end{aligned}
$$

for all arbitrary $\varepsilon \neq 0, \varepsilon \in R^{n}$, which verifies the property ( $\left.K_{1}\right)$ of Krasovskii [5].

Let $(x, y, z, w)=(x(t), y(t), z(t), w(t))$ be an arbitrary solution of the system (1.3). A straightforward calculation from (2.1) and (1.3) yields that

$$
\begin{aligned}
\dot{V}_{0}=\frac{d}{d t} V_{0}(x, y, z, w)= & \langle z, z\rangle+\langle x, G(x, y, z, w) z\rangle+\langle x, F(x, y, z, w) x\rangle \\
& +\langle H(x) y, x\rangle-\frac{d}{d t} \int_{0}^{1}\langle H(\sigma x) x, x\rangle d \sigma .
\end{aligned}
$$

Now, recall that

$$
\begin{aligned}
\frac{d}{d t} \int_{0}^{1}\langle H(\sigma x) x, x\rangle d \sigma & =\int_{0}^{1}\langle H(\sigma x) x, y\rangle d \sigma+\int_{0}^{1}\langle\sigma J(H(\sigma x) x \mid \sigma x) y, x\rangle d \sigma \\
& =\int_{0}^{1}\langle H(\sigma x) x, y\rangle d \sigma+\int_{0}^{1} \sigma\langle J(H(\sigma x) x \mid \sigma x) x, y\rangle d \sigma \\
& =\int_{0}^{1}\langle H(\sigma x) x, y\rangle d \sigma+\int_{0}^{1} \sigma \frac{\sigma}{\partial \sigma}\langle H(\sigma x), y\rangle d \sigma \\
& =\left.\sigma\langle H(\sigma x) x, y\rangle\right|_{0} ^{1}=\langle H(x) x, y\rangle .
\end{aligned}
$$

Substituting the estimate (2.3) into (2.2), we obtain

$$
\dot{V}_{0}=\langle z, z\rangle+\langle x, G(x, y, z, w) z\rangle+\langle x, F(x, y, z, w) x\rangle
$$


Now, it is clear that

$$
\dot{V}_{0}(0, y, z, w)=\langle z, z\rangle=\|z\|^{2} \geq 0 .
$$

Next, the function $\dot{V}_{0}$ can be rearranged as follows

$$
\begin{aligned}
\dot{V}_{0} & =\left\|z+\frac{1}{2} G(x, y, z, w) x\right\|^{2}+\langle x, F(x, y, z, w) x\rangle \\
& -\frac{1}{4}\langle G(x, y, z, w) x, G(x, y, z, w) x\rangle .
\end{aligned}
$$

Thus, we conclude that

$$
\dot{V}_{0} \geq\langle x, F(x, y, z, w) x\rangle-\frac{1}{4}\langle G(x, y, z, w) x, G(x, y, z, w) x\rangle>0
$$

because of the assumption of Theorem $1, \lambda_{i}(F(x, y, z, w))>\frac{1}{4}\left[\lambda_{i}(G(x, y, z, w))\right]^{2}$.

Hence, the above discussion clarifies that the function $\dot{V}_{0}$ is positive semi-definite. This case verifies the property $\left(K_{2}\right)$ of Krasovskii [5]. Next, let $(x(t), y(t), z(t), w(t))$ be an arbitrary solution of the system (1.3). We observe from the foregoing estimate of $\dot{V}_{0}$ that $\dot{V}_{0}(t)=0(t \geq 0)$ necessarily implies that $x(t)=0$ for all $t \geq 0$. In turn, this implies that

$$
y(t)=\dot{x}(t), z(t)=\dot{y}(t)=0, w(t)=\dot{z}(t)=0 \text { for all } t \geq 0 .
$$

Hence the property of $\left(K_{3}\right)$ of Krasovskii [5] holds for the function $V_{0}$.

This completes the proof of Theorem 1. form:

Example 1. In the system (1.3) in case of $n=2$ let $A, G$ and $F$ take the following

and

$$
\begin{aligned}
& A=\left[\begin{array}{ll}
4 & 2 \\
2 & 7
\end{array}\right], \\
& G=\left[\begin{array}{cc}
1+\frac{1}{1+x^{2}+y^{2}} & \frac{1}{1+x^{2}+y^{2}} \\
\frac{1}{1+x^{2}+y^{2}} & 1+\frac{1}{1+x^{2}+y^{2}}
\end{array}\right]
\end{aligned}
$$

$$
F=\left[\begin{array}{cc}
4+\frac{1}{1+x^{2}+y^{2}} & 0 \\
0 & 4+\frac{1}{1+x^{2}+y^{2}}
\end{array}\right]
$$

It is obvious that $A, G$ and $F$ are symmetric matrices. Then, by an easy calculation, we obtain eigenvalues of the matrices $A, G(x, y)$ and $F(x, y)$ as follows

and

$$
\begin{gathered}
\lambda_{1}(A)=3, \lambda_{2}(A)=8 \\
\lambda_{1}(G)=1, \lambda_{2}(G)=1+\frac{2}{1+x^{2}+y^{2}}
\end{gathered}
$$

$$
\lambda_{1}(F)=4+\frac{1}{1+x^{2}+y^{2}}, \lambda_{2}(F)=4+\frac{1}{1+x^{2}+y^{2}} .
$$

Clearly, all the conditions of Theorem 1 hold for arbitrary $H(x)$. Indeed, this fact shows that the trivial solution of corresponding vector differential equation for the 
above case $n=2$ is unstable. It should also be noted that, when $G$ and $F$ reduce to the linear case, our conclusions remain valid, too.

Our last result is the following theorem

Theorem 2. In addition to the basic assumptions imposed on $C, D, \Psi$ and $\Phi$ that appear in equation (1.2), we suppose that there exist constants $a_{3}$ and $a_{4}$ such that the following conditions hold:

$$
\lambda_{i}(C) \geq a_{3}>0, \lambda_{i}(D) \geq a_{4}>0
$$

and

$$
\lambda_{i}(D)>\frac{1}{4}\left[\lambda_{i}(\Psi(x, y, z, w))\right]^{2},(i=1,2, \ldots, n),
$$

for all $x, y, z, w \in R^{n}$.

Then the trivial solution $x=0$ of equation (1.2) is unstable for arbitrary $\Phi$ provided that $\Phi(0)=0$.

Remark 4. It should be noted that there exists no restriction on the vector field $\Phi$ in the system (1.4), except $J_{\Phi}(w)$ is symmetric.

Remark 5. Our second result, Theorem 2 includes the result of Ezeilo [3, Theorem 2] for the special case $n=1$ in the system (1.4), and improves the same result to the vector case. Equation (1.2) is also completely different from those investigated in the relevant literature, (see also Ezeilo [2-4], Sadek [7], Skrapek [8] and Tunç [9-11]. Remark 1 is also valid for Theorem 2.

Proof. For the proof of Theorem 2, we define a Lyapunov function $V_{1}=$ $V_{1}(x, y, z, w)$ given by:

$$
\begin{aligned}
V_{1}= & -\langle w, C y\rangle+\frac{1}{2}\langle C z, z\rangle-\langle D x, w\rangle+\langle D y, z\rangle \\
& -\int_{0}^{1}\langle\Phi(\sigma w), w\rangle d \sigma .
\end{aligned}
$$

We observe that $V_{1}(0,0,0,0)=0$. Indeed, in view of the assumptions of Theorem 2, we also have that

$$
\begin{aligned}
V_{1}(0,0, \varepsilon, 0) & =\frac{1}{2}\langle C \varepsilon, \varepsilon\rangle \\
& \geq \frac{a_{3}}{2}\langle\varepsilon, \varepsilon\rangle \\
& =\frac{a_{3}}{2}\|\varepsilon\|^{2}>0
\end{aligned}
$$

for all arbitrary $\varepsilon \neq 0, \varepsilon \in R^{n}$, which verifies the property ( $\left.K_{1}\right)$ of Krasovskii [5]. Next, let $(x, y, z, w)=(x(t), y(t), z(t), w(t))$ be an arbitrary solution of the system 
(1.4). A straightforward calculation from (2.4) and (1.4) gives that

$$
\begin{aligned}
\dot{V}_{1}=\frac{d}{d t} V_{1}(x, y, z, w)= & -\langle\dot{w}, C y\rangle-\langle\dot{w}, D x\rangle+\langle D z, z\rangle \\
& -\frac{d}{d t} \int_{0}^{1}\langle\Phi(\sigma w), w\rangle d \sigma .
\end{aligned}
$$

Now, recall that

$$
\frac{d}{d t} \int_{0}^{1}\langle\Phi(\sigma w), w\rangle d \sigma=\int_{0}^{1} \sigma\left\langle J_{\Phi}(\sigma w) \dot{w}, w\right\rangle d \sigma+\int_{0}^{1}\langle\Phi(\sigma w), \dot{w}\rangle d \sigma .
$$

But, $J_{\Phi}$ being symmetric,

$$
\left\langle J_{\Phi}(\sigma w) \dot{w}, w\right\rangle=\left\langle\dot{w}, J_{\Phi}(\sigma w) w\right\rangle
$$

so that

$$
\begin{aligned}
\int_{0}^{1} \sigma\left\langle J_{\Phi}(\sigma w) \dot{w}, w\right\rangle d \sigma & =\int_{0}^{1} \sigma\left\langle\dot{w}, J_{\Phi}(\sigma w) w\right\rangle d \sigma=\int_{0}^{1} \sigma \frac{d}{d \sigma}\langle\dot{w}, \Phi(\sigma w)\rangle d \sigma \\
& =\left.\sigma\langle\dot{w}, \Phi(\sigma w)\rangle\right|_{0} ^{1}-\int_{0}^{1}\langle\dot{w}, \Phi(\sigma w)\rangle d \sigma \\
& =\langle\dot{w}, \Phi(w)\rangle-\int_{0}^{1}\langle\dot{w}, \Phi(\sigma w)\rangle d \sigma .
\end{aligned}
$$

Hence,

$$
\frac{d}{d t} \int_{0}^{1}\langle\Phi(\sigma w), w\rangle d \sigma=\langle\dot{w}, \Phi(w)\rangle .
$$

Substituting the estimate (2.6) into (2.5), and then considering the system (1.4) and the assumptions of Theorem 2, we obtain

$$
\begin{aligned}
\dot{V}_{1}= & -\langle\dot{w}, \Phi(w)\rangle-\langle\dot{w}, C y\rangle-\langle\dot{w}, D x\rangle+\langle D z, z\rangle \\
= & \langle\dot{w}, \dot{w}\rangle+\langle\dot{w}, \Psi(x, y, z, w) z\rangle+\langle D z, z\rangle \\
= & \left\|\dot{w}+\frac{1}{2} \Psi(x, y, z, w) z\right\|^{2}+\langle D z, z\rangle \\
& -\frac{1}{4}\langle\Psi(x, y, z, w) z, \Psi(x, y, z, w) z\rangle
\end{aligned}
$$


Now, using the definition of $\dot{w}$ in (1.4), we get

$$
\begin{aligned}
\dot{V}_{1}= & \left\|\dot{w}+\frac{1}{2} \Psi(x, y, z, w) z\right\|^{2}+\langle D z, z\rangle \\
& -\frac{1}{4}\langle\Psi(x, y, z, w) z, \Psi(x, y, z, w) z\rangle \\
= & \left\|\Phi(w)+\frac{1}{2} \Psi(x, y, z, w) z+C y+D x\right\|^{2}+\langle D z, z\rangle \\
& -\frac{1}{4}\langle\Psi(x, y, z, w) z, \Psi(x, y, z, w) z\rangle .
\end{aligned}
$$

Clearly, it follows from (2.7) that

$$
\dot{V}_{1} \geq\langle D z, z\rangle-\frac{1}{4}\langle\Psi(x, y, z, w) z, \Psi(x, y, z, w) z\rangle .
$$

As a result, the above discussion and (2.7) provide that the function $\dot{V}_{1}$ is positive semi-definite because of assumption $\lambda_{i}(D)>\frac{1}{4}\left[\lambda_{i}(\Psi(x, y, z, w))\right]^{2}$ of Theorem 2 . This case verifies the property $\left(K_{2}\right)$ of Krasovskii [5].

Finally, let $(x(t), y(t), z(t), w(t))$ be an arbitrary solution of the system (1.4). We observe from the foregoing estimate of $\dot{V}_{1}$ that $\dot{V}_{1}(t)=0(t \geq 0)$ necessarily implies that $z(t)=0$ for all $t \geq 0$. In turn, this fact implies that

$$
w(t)=\dot{z}(t)=0 \text { for all } t \geq 0 .
$$

Since $\Phi(0)=0$ we have also

$$
C y(t)+D x(t)=0 \text { for all } t \geq 0,
$$

that is,

$$
C \dot{x}(t)+D x(t)=0 \text { for all } t \geq 0 .
$$

Because of $\ddot{x}(t)=0, \dot{x}(t)=c \in R^{n}$ for all $t \geq 0$. Hence by (2.8), since $D \neq 0$, we have that $x(t)=c \in R^{n}$ for all $t \geq 0$. But this implies that $\dot{x}(t)=0$ and thus also, by (2.8), that $x(t)=0$ for all $t \geq 0$.

This completes the verification of the property of $\left(K_{3}\right)$ of Krasovskii [5] for the function $V_{1}$.

Theorem 2 is thereby established.

\section{ACKNOWLEDGEMENT}

The author would like to expresses his sincere thanks to the referee for many helpful corrections and suggestions. 


\section{REFERENCES}

[1] R. Bellman, Introduction to matrix analysis, ser. Classics in Applied Mathematics. Philadelphia: Society for Industrial and Applied Mathematics (SIAM), 1997, vol. 19.

[2] J. O. C. Ezeilo, "An instability theorem for a certain fourth order differential equation," Bull. London Math. Soc., vol. 10, no. 2, pp. 184-185, 1978.

[3] J. O. C. Ezeilo, "Further instability theorems for some fourth order differential equations," $J$. Nigerian Math. Soc., vol. 19, pp. 1-7, 2000.

[4] J. O. C. Ezeilo, "Extension of certain instability theorems for some fourth and fifth order differential equations," Atti Accad. Naz. Lincei Rend. Cl. Sci. Fis. Mat. Natur. (8), vol. 66, no. 4, pp. 239-242, 1979.

[5] N. N. Krasovskii, "On conditions of inversion of a. m. lyapunov's theorems on instability for stationary systems of differential equations," Dokl. Akad. Nauk SSSR (N.S.), vol. 101, pp. 17-20, 1955.

[6] A. M. Liapunov, Stability of motion, ser. Mathematics in Science and Engineering. New YorkLondon: Academic Press, 1966, vol. 30.

[7] A. I. Sadek, "Instability results for certain systems of fourth and fifth order differential equations," Appl. Math. Comput., vol. 145, no. 2-3, pp. 541-549, 2003.

[8] W. A. Skrapek, "Instability results for fourth-order differential equations," Proc. Roy. Soc. Edinburgh Sect. A, vol. 85, no. 3-4, pp. 247-250, 1980.

[9] C. Tunç, "An instability theorem for a certain vector differential equation of the fourth order," JIPAM. J. Inequal. Pure Appl. Math., vol. 5, no. 1, p. 5, 2004.

[10] C. Tunç, "A further instability result for a certain vector differential equation of fourth order," Int. J. Math. Game Theory Algebra, vol. 15, no. 5, pp. 489-495, 2006.

[11] C. Tunç, "Instability of solutions for certain nonlinear vector differential equations of fourth order," Nonlinear Oscil. (N. Y.), vol. 12, no. 1, pp. 123-132, 2009.

\section{Author's address}

\section{Cemil Tunç}

Yüzüncü Yll University, Department of Mathematics, Faculty of Arts and Sciences, 65080, Van, Turkey

E-mail address: cemtunc@yahoo.com 